\title{
Influence of ion-induced interfacial chemical reactivity on contact resistance
}

\author{
S T LAKSHMIKUMAR \\ National Physical Laboratory, Dr K S Krishnan Road, New Delhi 110012, India \\ MS received 5 December 1994; revised 29 March 1995
}

\begin{abstract}
Contact resistance measurements of chromium contacts deposited by partially jonized beam deposition on transparent conducting indium tin oxide (ITO) were performed. These provide a direct experimental evidence of the influence of interfacial chemical interaction on the contact resistance. The interfacial reactivity is controlled by modifying the energy and flux of ionized chromium atoms deposited on ITO employing a specially designed partially ionized deposition system with very high ionization efficiency. The true contact resistivity $\rho_{\mathrm{c}}$ is obtained by iteratively correcting the experimentally measured values for the finite sheet resistance of the ITO layer. $\rho_{c}$ decreases linearly with the energy of the ionized chromium. Auger sputter profiling shows no structural modifications at the interface due to a change in the energy of the chromium atoms, confirming that the observed change in the contact resistivity is directly related to interfacial chemical bonding of the atoms with the oxygen atoms in the ITO leading to a local increase of carrier concentration and lower interfacial resistance.
\end{abstract}

Keywords. Chromium contacts; beam deposition.

\section{Introduction}

Contact resistance, the resistance to flow of electric current at the interface between two materials, is a major technological problem, particularly when large current densities are encountered. In microelectronic devices with very small contact areas, the crowding of current lines and the electronic band structure of the ohmic contact contribute to the contact resistance (Cohen and Gildenblat 1986). Contact resistance is also an important technological problem in solar cells (Hovel 1975; Kanicki 1988; Rastogi and Lakshmikumar 1989). In addition, our work on metal oxide conductor contacts, where oxygen has a direct role to play in the generation of the conducting species, has shown that the physicochemical interactions at the interface directly control the contact resistance. Two distinct processes with different physical origins contribute to the contact resistance (Lakshmikumar and Rastogi 1989). One relates to the modification in the detailed charge balance and carrier concentration at the interface induced by the proximal contact metal. The second is due to the nature of carrier injection at the free surface of the oxide conductor. This was confirmed by the observed scaling of $\rho_{\mathrm{c}}$ with the strength of metal-oxygen interaction represented by the heat of formation of the oxide. The interaction between oxygen and metal leads to the modification of the availability of conducting species at the interface.

Partially ionized deposition is a powerful technique for the controlled variation of the reactivity of adatoms. Ion bombardment is known to influence adhesion, stress, impurity content and packing density (McNiel et al 1984). Low-energy ion bombardment (energy $\leqslant 200 \mathrm{eV}$ ) has the capacity to provide activation energy for the growth of epitaxial films (Takagi et al 1975; Itoh et al 1977; Appleton et al 1986), to significantly affect the growth process in thin films (Harper et al 1983), to modify the interfacial 
electronic properties of Schottky barriers (Wong et al 1986) and to enhance surface reconstruction and reaction leading to the formation of compounds at very low temperatures ( $\mathrm{Li}$ and Lu 1990; Liu et al 1993). At the same time, the total ionic energy is small and there is no structural damage to the interface between the substrate and the film (Greene et al 1986).

The present work is an attempt to directly modify the reactivity of a depositing species and study the resultant changes in contact resistivity. The special design of the electron bombardment heater provides high concentrations, up to $5 \%$, of ions (Mei and Lu 1988). By biasing the substrate, the energy and flux of the ions impinging on the substrate can be directly modified, thereby controlling the interfacial reactivity. Chromium was selected as the contact metal because $\mathrm{Cr} / \mathrm{ITO}$ contacts show the lowest resistivities. The contact resistance on ITO was measured by depositing chromium contacts with various fluxes of the ionized species in a 4-terminal contact resistivity test structure configuration (Chern and Oldhiem 1984). The true contact resistivity, $\rho_{\mathrm{c}}$ was iteratively calculated from the experimentally measured resistance. Auger sputter profiling has been employed to determine the width of the interface and the bonding state of the chromium atoms.

\section{Experimental}

Chromium films were deposited using partially ionized beam (PIB) deposition procedure described earlier (Mei and $\mathrm{Lu} \mathrm{1988).} \mathrm{Cr} \mathrm{metal} \mathrm{is} \mathrm{evaporated} \mathrm{from} \mathrm{a} \mathrm{graphite}$ crucible by heating with an electron beam accelerated to a high positive potential $(\approx 1 \mathrm{kV})$ applied to the crucible. A tantalum filament is used to produce a high $(\approx 1 \mathrm{~A})$ electron current by thermionic emission. The unique filament design results in $2-5 \%$ ionization as determined from the ion current measurements. This high level of ionization for an indirect ionization procedure is primarily due to the extremely high electron density in the vicinity of the mouth of the graphite crucible which enhances the probability of atom-electron collisions. The system has a base pressure of $10^{-5}$ $\mathrm{Pa}$. An external retarding bias is applied to the substrate to contiol the energy of the ionized beam, as required. The ITO substrates were initially cleaned in alkaline detergent and then washed in hot running water. They were then degreased in boiling trichloroethylene before loading into the system through a load-lock arrangement. The $\mathrm{Cr}$ metal ( $4 \mathrm{~N}$ purity) was evaporated at a rate of $\approx 1 \AA / \mathrm{s}$ at a background pressure of $\approx 3 \times 10^{-5} \mathrm{~Pa}$, to a final thickness of $1000 \AA$. Depositions were performed at various bias voltages from $0 \mathrm{~V}$ (ion energy $\approx 1 \mathrm{keV}$ ) to $1 \mathrm{kV}$ (ion energy $\approx 0 \mathrm{eV}$ ). The substrates were not heated and the temperature rise due to incidental heating from the source was measured to be less than $20^{\circ} \mathrm{C}$. For contact resistance measurements, the contacts were formed on the freshly cleaned ITO of sheet resistance $20-30 \Omega / \mathbf{a}$ by evaporating the metal through lithographically delineated masks. The wide contact test structure (Chern and Oldhiem 1984) which is most appropriate for large-area contacts was employed for the contact resistivity measurements. Typical contact area was $\approx 0.01 \mathrm{~cm}^{2}$. The schematic view of the wide contact test structure employed is shown in figure 1. The experimental contact resistance $R_{\text {exp }}$ was determined by four-probe measurement using the $I-V$ leads as shown in figure 1 . The contribution from the ITO layer under the contact can be evaluated by invoking the transmission line model, and the true contact resistivity $\rho_{\mathrm{e}}$ determined (Chern and Oldhiem 1984) 


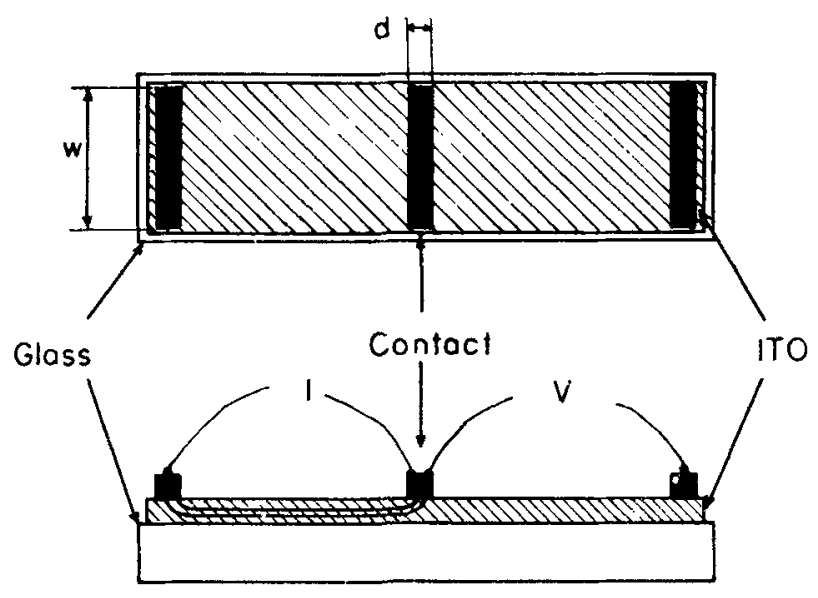

Figure 1. Schematic of the wide contact test structure.

iteratively using the equation

$$
R_{\exp }=\left(R_{\mathrm{s}} \rho_{\mathrm{c}}\right)^{1 / 2} /\left\{w \sinh \left[\left(R_{\mathrm{s}} / \rho_{\mathrm{c}}\right)^{1 / 2} \cdot d\right]\right\}
$$

Here $R_{\mathrm{s}}$ is the sheet resistance of ITO, measured individually for each test structure, and $w$ and $d$ are the dimensions of the contact determined by optical microscopy for each contact. In view of the low temperatures involved in the preparation of the contact, it was assumed that $R_{\mathrm{s}}$ is not altered during the process of deposition of the metal layer. AES was performed on samples for evaluating the quality of the films and the interface. For depth profile studies, the film was sputtered away using $3 \mathrm{kV} \mathrm{Ar}$ ions. The composition was evaluated using the height of the $532 \mathrm{eV}$ peaks for $\mathrm{Cr}$ since the main peak overlaps with the oxygen peak at $515 \mathrm{eV}$. The composition of the ITO was evaluated by monitoring the lines of $\operatorname{In}(400 \mathrm{eV}), \operatorname{Sn}(428 \mathrm{eV})$ and $\mathrm{O}_{2}(515 \mathrm{eV})$ respectively.

\section{Results and discussion}

The variation of the contact resistivity with the applied bias voltage is shown in figure 2 . The experimentally measured contact resistance $R_{\exp }$ and the true contact resistance (the product of the iterated contact resistivity $\rho_{\mathrm{c}}$ and the experimental contact area $w \cdot d)$ are shown. Both decrease as the ion energy increases. The values of $R_{\text {exp }}$ and $R_{\mathrm{c}}$ shown are averaged over several samples. The scatter in data is $\approx 10 \%$. The parasitic contribution, the difference between the measured contact resistance $R_{\text {exp }}$ and the true contact resistance $\left(\rho_{c} \cdot w \cdot d\right)$ is small for the low-sheet-resistance ITO employed in this study. All the contacts were completely ohmic over a current range of $10^{-6}-2 \times 10^{-1}$ A, ruling out the presence of interfacial insulating layers. The values of $R_{\mathrm{c}}$ obtained are similar to earlier measurements on thermally deposited $\mathrm{Cr}$ films. The lowest value obtained is $1.51 \times 10^{-2} \Omega \mathrm{cm}^{2}$, less than the lowest value previously reported for $\mathrm{Cr}$ contacts on $10 \Omega /$ ITO. For ITO with similar sheet resistances, earlier data show a value of $\approx 6 \times 10^{-2} \Omega \mathrm{cm}^{2}$ which is very much higher. When high bias is used to 


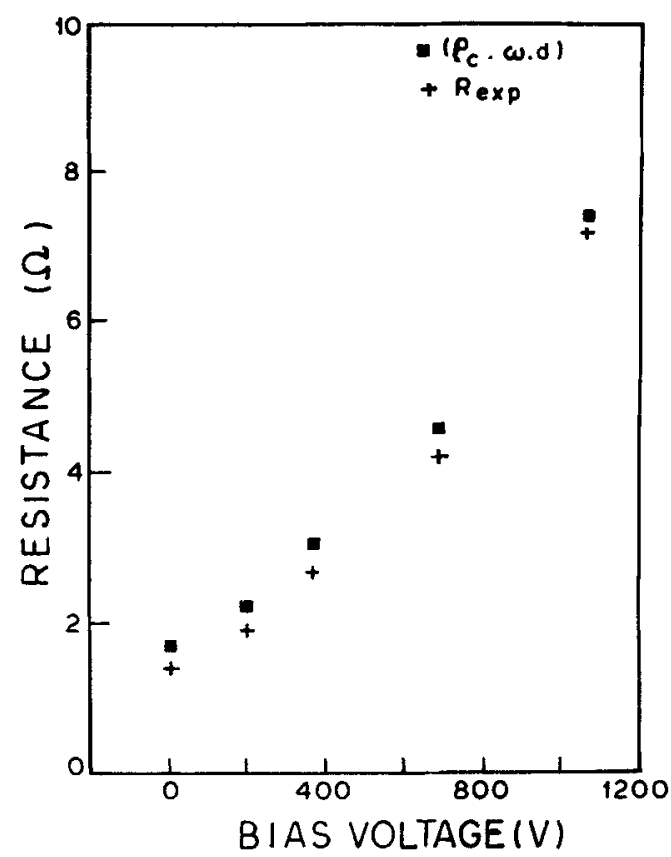

Figure 2. Variation of the contact resistance with the ion energy.

ensure that no ionized species are being deposited, the $\rho_{\mathrm{c}}$ value is $6.4 \times 10^{-2} \Omega \mathrm{cm}^{2}$, which is comparable to the earlier values obtained for thermally deposited contacts.

The contact resistivity in these conducting oxides is not determined by the semiconductor contact theory (Chang et al 1971), which predicts

$$
\rho_{\mathrm{c}} \approx \exp \left(V_{\mathrm{bo}} / E_{\mathrm{oo}}\right)
$$

Here, $V_{\text {bo }}$ is the barrier height between ITO and the metal and $E_{\mathrm{oo}}$ is an energy term which depends on the effective mass, $m^{*}$ and density $N$ of the carriers and the effective permittivity of the semiconductor, $\varepsilon$, as

$$
1 / E_{\mathrm{oo}}=(h / 2)\left(N / m^{*} \varepsilon\right)^{1 / 2} .
$$

It is difficult to estimate the theoretical value of $\rho_{c}$, since the constants are unknown. However, ITO has an effective carrier concentration of $\approx 10^{21}$, and at these densities, calculations on Si show that the value is very low $\left(\approx 10^{-7}\right)$ and is generally insensitive to other parameters.

It is clear that the variation of $R_{\mathrm{c}}$ with the effective ion energy is only explained in terms of the model developed earlier (Lakshmikumar and Rastogi 1989) based on the physicochemical interactions at the interface. In terms of this model, the effective ion energy of the $\mathrm{Cr}$ atoms increases the binding between the $\mathrm{Cr}$ and $\mathrm{O}$ atoms at the interface and this interaction directly controls the interfacial resistance. Since the oxygen vacancies act as double donors in ITO, the freezing of the oxygen atoms at the interface would effectively alter the carrier density and hence the $\rho_{\mathrm{c}}$. Unlike earlier 
work, the present studies involve a single metal and the reactivity has been experimentally modified.

In order to rule out the possibility of structural modification of the interface due to the penetration of ionized $\mathrm{Cr}$ atoms, Auger sputter studies were performed on some samples. The typical compositional profile at the interface is shown in figure 3 . The width of the interface, estimated from the time taken to sputter known thicknesses of $\mathrm{Cr}$ and ITO, is $\approx 100 \AA$. The shape of the $\mathrm{Cr}$ Auger line at $530 \mathrm{eV}$ at various depths in the interface region is shown as an inset in figure 3. This line shows a distortion as the interface is traversed. The $\mathrm{Cr}$ line observed at the $\mathrm{Cr}$ side of the interface, at points $\mathrm{A}$ and $\mathrm{B}$ in figure 3 , shows free $\mathrm{Cr}$. The $\mathrm{Cr}$ in intimate contact with the ITO appears to be bonded. This is clear from the line shape of the $530 \mathrm{eV}$ line at position $\mathrm{C}$ in figure 3.

The shape of the sputter profile, width of the interface region, and the lineshape of the $\mathrm{Cr}$ Auger line do not change with the energy of the ions. The variation in the experimentally determined width of the interface with ion energy is not significant. The bonded $\mathrm{Cr}$ is located only after the interfacial region is sputtered away. The width of the region showing bonded $\mathrm{Cr}$ (the region beyond the position $C$ in figure 3 ) is $\approx 100 \AA$ at all ion energies. These results suggest that there is no systematic increase of the penetration of the adatoms into ITO as the ion energy increases from zero to $\approx 1 \mathrm{keV}$. This confirms that the observed variation of contact resistivity with the ion energy is not related to structural changes involving the penetration of ions into ITO. The observed local bonding of $\mathrm{Cr}$ supports our model of interfacial resistance being controlled by physicochemical interactions at the contact.

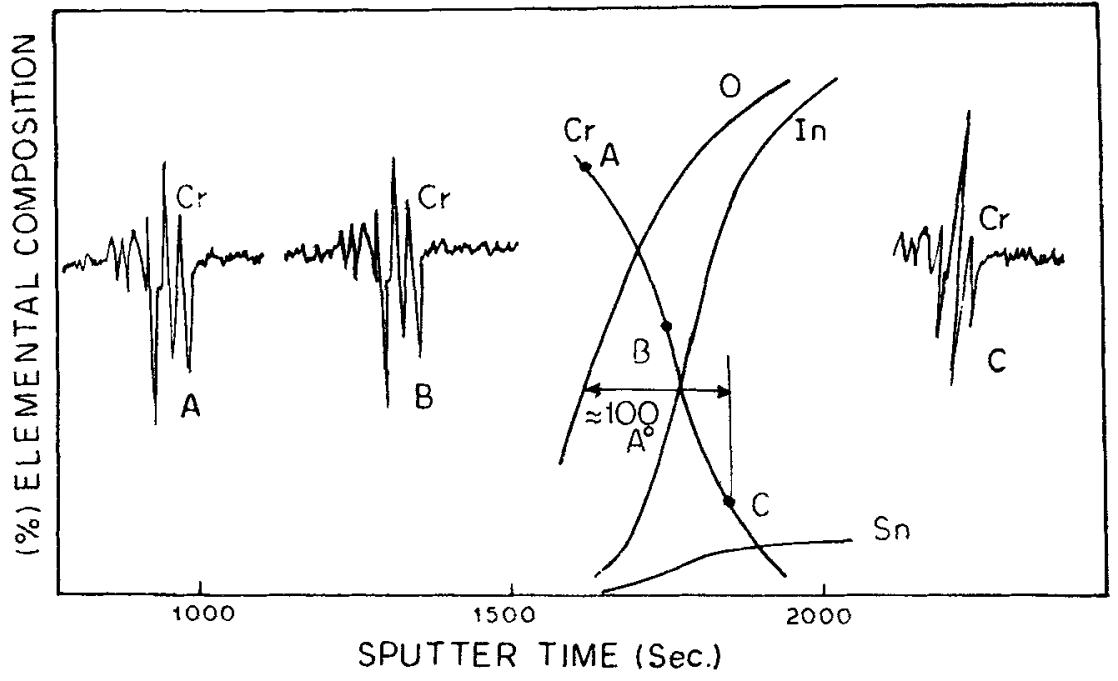

Figure 3. Auger sputter profile of the $\mathrm{Cr} / \mathrm{ITO}$ interface. The inset shows the change in the lineshape of the $\mathrm{Cr}$ Auger line at $530 \mathrm{eV}$ at the interface. 


\section{Conclusions}

The interfacial resistivity of $\mathrm{Cr} / \mathrm{ITO}$ contacts formed by partially ionized deposition of the metal have been measured. The resistivity decreases with the increase in the effective ion energy. All the contacts were completely ohmic over a current range of $10^{-6}-2 \times 10^{-1} \mathrm{~A}$. The lowest value obtained is $1.51 \times 10^{-2} \Omega \mathrm{cm}^{2}$, less than the lowest values reported in earlier measurements on thermally deposited $\mathrm{Cr}$ films on $10 \Omega /$ a ITO. Auger studies show no systematic variation of the interfacial depth or structural changes at the interface as the ion energy is varied. Lineshape analysis of the $530 \mathrm{eV}$ Auger line of $\mathrm{Cr}$ shows that the $\mathrm{Cr}$ atom becomes bonded at the interface. These results show that the observed variation of interfacial resistivity is not due to structural modifications at the interface and provide a direct evidence for the influence of chemical interactions at the interface on contact resistivity.

\section{Acknowledgements}

The author thanks Prof. T M Lu, Centre for Integrated Electronics, Rensselaer Polytechnic Institute, New York, where the depositions were performed during his stay there as a Raman Fellow. Thanks are due to Dr S M Shivaprasad for the Auger measurements.

\section{References}

Appleton B R, Zuhr R A, Noggle T S, Herbots N and Pennycook S J 1986 MRS Sym. Proc. Photon, beam and plasma stimulated chemical processes at surfaces (eds) V M Donnelly, I P Hermann and M Hirose (New York: MRS) p 319

Chern J G J and Oldhiem W G 1984 IEEE Electron Dev. Lett. 5178

Cohen S S and Gildenblat G S 1986 VLSI Science and Technology (ed.) N G Einspruch 13 p. 87

Greene J E, Rockett A and Sundgren J E 1986 MRS Sym. Proc. Photon, beam and plasma stimulated chemical processes at surfaces (eds) V M Donnelly, I P Hermann and M Hirose (New York: MRS) p. 39

Harper J M E, Cumo V J and Kaufmann H R 1983 Ann. Reviews of Mater. Sci. (eds) R A Huggins, R H Bube and D A Vermilea 13413

Hovel H J 1975 Semiconductors and semi-metals (eds) R K Willardson and A C Beer 11 p. 1

Itoh T, Nakamura T, Utomachi M and Sugiyama T 1977 Jap. J. Appl. Phys. 16553

Kanicki J 1988 Appl. Phys. Lett. 531943

Lakshmikumar S T and Rastogi A C 1989 Appl. Phys. A48 325

Li P and Lu T M 1990 Appl. Phys. Lett. 572336

Liu W T, Lakshmikumar S T, Knorr D B, Lu T M and van der Leeden Ir G A 1993 Appl. Phys. Lett. 64573

McNiel J R, Barron A C, Wilson S R and Hermann W C Jr. 1984 Appl. Opt. 23552

Mei S N and Lu T M 1988 J. Vac. Sci. Technol. A69

Rastogi A C and Lakshmikumar S T 1989 Solar Cells 26323

Takagi T, Tamada I and Sasaki A 1975 Thin Solid Films 45569

Wong J, Mei S N and Lu T M 1986 in MRS Sym. Proc. Interfaces, superlatices and thin films (eds) J D Dow and I K Schuller (New York: MRS) p. 211 